suggests ZIKV as the primary flavivirus infection. The limited antibody response in Patient 2 was presumably due to her ongoing immunosuppressive therapy. Although neither patient reported symptoms associated with ZIKV infection during the investigation, these data show evidence for ZIKV transmission by means of platelet transfusion.

lara J.F. Motta, M.D.

Instituto Nacional de Câncer José Alencar Gomes da Silva Rio de Janeiro, Brazil

Bryan R. Spencer, M.P.H.

American Red Cross, Massachusetts Region Dedham, MA

Orlando C. Ferreira, Jr., M.D., Ph.D.

Universidade Federal do Rio de Janeiro

Rio de Janeiro, Brazil

orlandocfj@gmail.com

and Others
Dr. Motta and Mr. Spencer contributed equally to this letter. A complete list of authors is available with the full text of this letter at NEJM.org.

Disclosure forms provided by the authors are available with the full text of this letter at NEJM.org.

This letter was published on August 17, 2016, at NEJM.org.

1. Foy BD, Kobylinski KC, Chilson Foy JL, et al. Probable nonvector-borne transmission of Zika virus, Colorado, USA. Emerg Infect Dis 2011;17:880-2.

2. Musso D, Roche C, Robin E, Nhan T, Teissier A, Cao-Lormeau VM. Potential sexual transmission of Zika virus. Emerg Infect Dis 2015;21:359-61.

3. Besnard M, Lastere S, Teissier A, Cao-Lormeau V, Musso D. Evidence of perinatal transmission of Zika virus, French Polynesia, December 2013 and February 2014. Euro Surveill 2014; 19(13):pii:20751.

4. Musso D, Nhan T, Robin E, et al. Potential for Zika virus transmission through blood transfusion demonstrated during an outbreak in French Polynesia, November 2013 to February 2014. Euro Surveill 2014;19(14):pii:20761.

5. Lanciotti RS, Lambert AJ, Holodniy M, Saavedra S, Signor Ldel C. Phylogeny of Zika virus in Western Hemisphere, 2015. Emerg Infect Dis 2016;22:933-5.

DOI: 10.1056/NEJMc1607262

\title{
Treatment Outcomes in Multidrug-Resistant Tuberculosis
}

TO THE EDITOR: Despite lengthy treatment with costly second-line drug regimens, curing multidrug-resistant (MDR) tuberculosis (bacillary resistance to at least isoniazid and rifampin) remains a challenge. ${ }^{1}$ The World Health Organization (WHO) defines "cure" as "treatment completion" with at least three negative cultures after the intensive phase of therapy in the absence of "treatment failure." The definition of "treatment failure" requires early termination of treatment or the need for permanent regimen change of at least two antituberculosis drugs. "Treatment success" is defined as the sum of cure and treatment completion. ${ }^{2}$

We evaluated treatment outcomes according to WHO definitions in the TBNET cohort of 380 patients with MDR tuberculosis at 23 European centers, including 89 patients with pre-extensively drug-resistant (XDR) tuberculosis and 33 patients with XDR tuberculosis, ${ }^{3,4}$ and compared them with simplified definitions of MDR tuberculosis treatment outcomes (see the Supplementary Appendix, available with the full text of this letter at NEJM.org). Cure was defined as a negative culture status 6 months after treatment initiation, no positive culture thereafter, and no relapses within 1 year after treatment completion. Treat- ment failure was defined as a positive culture status 6 months after treatment initiation or thereafter or a relapse within 1 year after treatment completion. An undeclared outcome was defined as an outcome that was not assessed (owing to transferral out of the cohort, no culture status at 6 months while the patient was receiving care, or no post-treatment assessment). Death was defined as death during observation. Loss to follow-up was defined as nonreceipt of care 6 months after treatment initiation.

Fifty of 88 patients with treatment failure (57\%) were not identified by the WHO definition. Assessment of WHO-defined cure was possible for only $13 \%$ of the patients in countries with a low incidence of tuberculosis (with a notification rate $<20$ per 100,000 population), $58 \%$ of the patients in countries with an intermediate incidence (with a notification rate of 20 to 100 per 100,000 population), and $52 \%$ of the patients in countries with a high incidence (with a notification rate $>100$ per 100,000 population), owing to a lack of sputum cultures obtained after the intensive-treatment phase. This could reflect the limited access to health care of mostly foreignborn patients or the inability of patients to produce sputum in the latter stages of therapy. 


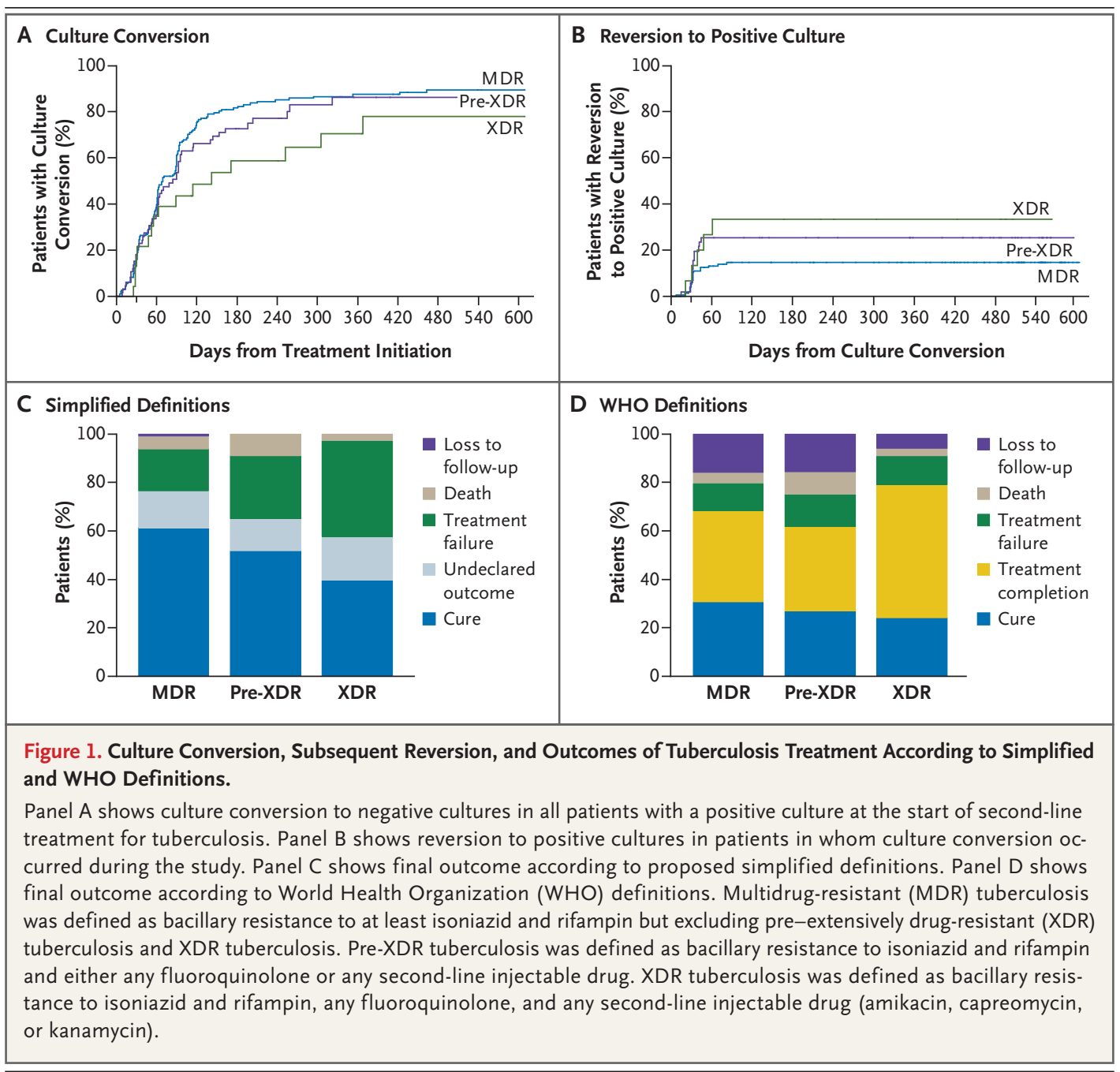

WHO-defined treatment success in MDR tuberculosis is predominantly driven by completing treatment rather than by a biologic end point and fails to address relapse-free survival as a clinically more relevant assessment of treatment efficacy.

Relapse-free cure was achieved in $61 \%$ of the patients with MDR tuberculosis, 52\% of the patients with pre-XDR tuberculosis, and 39\% of the patients with XDR tuberculosis when simplified definitions were used, in contrast to WHOdefined cure in $31 \%, 27 \%$, and $24 \%$ of patients, respectively (Fig. 1).

Of the 318 patients with a negative culture status at 6 months, 35 (11\%) reverted in the continuation phase, and 9 (3\%) had a posttreatment relapse, findings that suggest that culture status at 6 months may be a reliable predictor of relapse-free cure in patients with MDR tuberculosis. 5

In conclusion, current WHO definitions may underestimate cure in patients with MDR tuberculosis. These definitions could be simplified while incorporating the assessment of posttreatment relapse.

Gunar Günther, M.D., M.P.H. Christoph Lange, M.D., Ph.D.

Research Center Borstel

Borstel, Germany

clange@fz-borstel.de

Sofia Alexandru, M.D.

Institute of Phtisiopneumology

Chisinau, Moldova

Neus Altet, M.D.

Hospital Universitari Vall d'Hebron

Barcelona, Spain 
Korkut Avsar, M.D., Ph.D.

Asklepios Klinik Gauting

Gauting, Germany

Didi Bang, M.D., Ph.D.

Statens Serum Institut

Copenhagen, Denmark

Raisa Barbuta, M.D.

Balti Municipal Hospital

Balti, Moldova

Graham Bothamley, M.D., Ph.D.

Homerton University Hospital

London, United Kingdom

Ana Ciobanu, M.D.

Valeriu Crudu, M.D., Ph.D.

Institute of Phtisiopneumology

Chisinau, Moldova

Manfred Danilovits, M.D.

Tartu University Lung Hospital

Tartu, Estonia

Martin Dedicoat, M.D., Ph.D.

Heart of England Foundation Trust

Birmingham, United Kingdom

Raquel Duarte, M.D., Ph.D.

Porto University

Porto, Portugal

Gina Gualano, M.D.

Lazzaro Spallanzani National Institute for Infectious Diseases Rome, Italy

Heinke Kunst, M.D.

Queen Mary University Hospital

London, United Kingdom

Wiel de Lange, M.D.

University Medical Center Groningen

Groningen, the Netherlands

Vaira Leimane, M.D.

Riga East University Hospital

Riga, Latvia

Cecile Magis-Escurra, M.D., Ph.D.

Radboud University Medical Center

Nijmegen, the Netherlands

Anne-Marie McLaughlin, M.D.

St. James's Hospital

Dublin, Ireland

Inge Muylle, M.D.

University Medical Center St. Pieter

Brussels, Belgium

Veronika Polcová, M.D.

Thomayer University Hospital

Prague, Czech Republic

Christina Popa, M.D.

Marius-Nasta-Institut

Bucharest, Romania
Rudolf Rumetshofer, M.D.

Otto Wagner Hospital

Vienna, Austria

Alena Skrahina, M.D.

Varvara Solodovnikova, M.D.

Republican Research and Practical Center for Pulmonology

and Tuberculosis

Minsk, Belarus

Victor Spinu, M.D.

Marius-Nasta-Institut

Bucharest, Romania

Simon Tiberi, M.D.

Barts Health NHS Trust

London, United Kingdom

Piret Viiklepp, M.D.

National Institute for Health Development

Tallinn, Estonia

Frank van Leth, M.D., Ph.D.

Amsterdam Institute for Global Health and Development

Amsterdam, the Netherlands

for TBNET

Funded by the European Commission Seventh Framework Program (FP7/2007-2013) under grant agreement FP7-223681 (TB-PANNET). Dr. Lange is supported by the German Center for Infection Research (DZIF).

Disclosure forms provided by the authors are available with the full text of this letter at NEJM.org.

1. European Centre for Disease Prevention and Control/WHO Regional Office for Europe. Tuberculosis surveillance and monitoring in Europe 2015. Geneva: World Health Organization, 2015.

2. Definitions and reporting framework for tuberculosis 2013 revision. Geneva: World Health Organization, 2013.

3. Günther G, van Leth F, Alexandru S, et al. Multidrug-resistant tuberculosis in Europe, 2010-2011. Emerg Infect Dis 2015; 21:409-16.

4. Günther G, van Leth F, Altet N, et al. Beyond multidrug-resistant tuberculosis in Europe: a TBNET study. Int J Tuberc Lung Dis 2015;19:1524-7.

5. Kurbatova EV, Cegielski JP, Lienhardt C, et al. Sputum culture conversion as a prognostic marker for end-of-treatment outcome in patients with multidrug-resistant tuberculosis: a secondary analysis of data from two observational cohort studies. Lancet Respir Med 2015;3:201-9.

DOI: 10.1056/NEJMc1603274

Correspondence Copyright @ 2016 Massachusetts Medical Society.

INSTRUCTIONS FOR LETTERS TO THE EDITOR

Letters to the Editor are considered for publication, subject to editing and abridgment, provided they do not contain material that has been submitted or published elsewhere.

Letters accepted for publication will appear in print, on our website at NEJM.org, or both.

Please note the following:

- Letters in reference to a Journal article must not exceed 175 words (excluding references) and must be received within 3 weeks after publication of the article.

N ENGLJ MED 375;11 NEJM.ORG SEPTEMBER 15, 2016

The New England Journal of Medicine

Downloaded from nejm.org on January 30, 2017. For personal use only. No other uses without permission. 